\title{
http://revistainvestigacionacademicasinfrontera.com
}

Recibido el 20 de octubre de 2017 dictaminado mediante arbitraje favorablemente 28 de diciembre de 2017

\section{Modelo de expectativas en torno a la seguridad pública en microempresarios del centro de México.}

\author{
María Luisa Quintero Soto, Tirso Javier Hernández Gracia, Arturo Sánchez \\ Sánchez, Héctor Daniel Molina Ruíz, Cruz García Lirios
}

Resumen -La seguridad organizacional ha sido propuesta como un constructo para dar cuenta de la importancia de la prevención del delito en las empresas cuyo valor radica en la creación del conocimiento. En tal sentido es que el objetivo del presente trabajo fue explicar las dimensiones de la seguridad organizacional. Se realizó un estudio no experimental con una selección no probabilística de 258 microempresarios del centro de México. A partir de un modelo estructural $\left\lceil\mathrm{X}^{2}=324,35(21 \mathrm{gl}) \mathrm{p}=0,007 ; \mathrm{GFI}=0,990 ; \mathrm{CFI}=0,995 ; \mathrm{RMSEA}=0,009\right\rfloor$ se encontró que el factor relativo a la seguridad tecnológica esperada reflejó al constructo de seguridad organizacional (0,731).

Palabras claves -Globalización, localidad, modelo, intangibles, capital intelectual

\begin{abstract}
Organizational security has been proposed as a construct to account for the importance of crime prevention in companies whose value lies in the creation of knowledge. In this sense, the objective of this paper was to explain the dimensions of organizational security. A non-experimental study was carried out with a non-probabilistic selection of 258 microentrepreneurs from central Mexico. From a structural model $\lceil X 2=324.35$ (21gl) $p=0.007 ; G F I=0.990 ; C F I=$ 0.995; RMSEA = 0.009 $\mathrm{J}$ it was found that the factor related to the expected technological safety reflected the organizational safety construct (0.731).
\end{abstract}

Keywords -Globalization, locality, model, intangibles, intellectual capital

\section{Introducción}

El proyecto se inscribe en la disciplina de Trabajo Social, área de estudios documentales, pero incluye conceptos relativos a la psicología del delito, la sociología de la violencia y la economía de la seguridad.
Precisamente, el objetivo del presente trabajo es especificar un modelo para el estudio de las expectativas de la seguridad pública en microempresarios del centro de México, estableciendo la confiabilidad y la validez de un instrumento que mide las expectativas, considerando tres dimensiones relativas a los 


\section{http://revistainvestigacionacademicasinfrontera.com}

riesgos y beneficios de invertir en seguridad reclutadora, tecnológica e interpersonal.

De esta manera, la seguridad pública desde la psicología del delito es resultado de la sumatoria de intenciones y acciones orientadas a la comisión de un delito que dañe o lesione los intereses de terceras personas en cuanto a su patrimonio, estabilidad o integridad, pero tal aproximación soslaya el contexto de legitimación de la violencia que abre la discusión en torno a la naturaleza del delito como inherente a la genética humana o como resultado de la privación de recursos para un desarrollo personal, grupal o social.

Sin embargo, la perspectiva sociológica de la violencia no aclara si una vez descartada la versión genética las oportunidades no estarían determinando la comisión de un delito, principalmente el que consiste en el daño a la propiedad ajena, los fraudes o extorsiones a personas que por su función empresarial su protección y seguridad es de interés público.

De esta manera es que la seguridad pública, desde la óptica integradora del Trabajo Social radica en el equilibrio entre las demandas y oportunidades del entorno con respecto a las capacidades y los recursos de de los actores políticos y sociales, públicos y civiles con respecto a la prevención del delito y la impartición de justicia.

\section{Gobernanza de la seguridad pública empresarial}

La seguridad pública empresarial refiere a la presunta ocurrencia de delitos registrados en averiguaciones previas iniciadas, o carpetas de investigación, reportadas por las Procuradurías Generales de Justicia y Fiscalías Generales de las 32 entidades federativas, quienes son responsables de la veracidad y actualización de los datos. (Sistema de Seguridad Pública, 2015)

Vinculada a la noción de seguridad pública, la reinserción supone la generación de nuevos sistemas de creencias en los ex convictos, al considerar que sólo de esa manera se puede llegar a aceptar, y por ende llevar a cabo, conductas socialmente aprobadas por parte de los individuos (Vázquez, 2014).

En el ámbito organizacional, la seguridad pública empresarial y la captación de recursos humanos circunscritos a la reinserción laboral configuran un clima de relaciones conocido como formación profesional que tiene su correlato en el estrés, el mobbing o el stalking en el caso de protocolos digitales.

De este modo, la violencia formativa está indicada por relaciones asimétricas e inequitativas entre los integrantes de la red, una gestión sustituida por dogmas; las libertades desplazadas por la discrecionalidad; las oportunidades por las 


\section{http://revistainvestigacionacademicasinf rontera.com}

imposiciones, las capacidades reducidas a su mínima expresión frente a los parentescos y las corresponsabilidades son inhibidas por atribuciones de culpabilidad (Carreón, 2016).

Por consiguiente, el sistema de gestión y administración de las responsabilidades entre agresores y víctimas al interior de las organizaciones es conocido como gobernanza. Se trata de un nuevo estilo de gobierno, distinto del modelo de control jerárquico y de mercado, caracterizado por un mayor grado de cooperación entre los gobiernos y administraciones públicas y actores no gubernamentales en la hechura de las políticas públicas. Se espera que a través de este proceso de elaboración de las políticas, fundamentado en la colaboración, el consenso y la participación de distintos actores, se mejoren los resultados y rendimientos de las políticas y, en definitiva, se garantice la gobernabilidad del sistema político (Zurbriggen, 2011).

Figura 1. Participación civil para la incidencia en políticas públicas

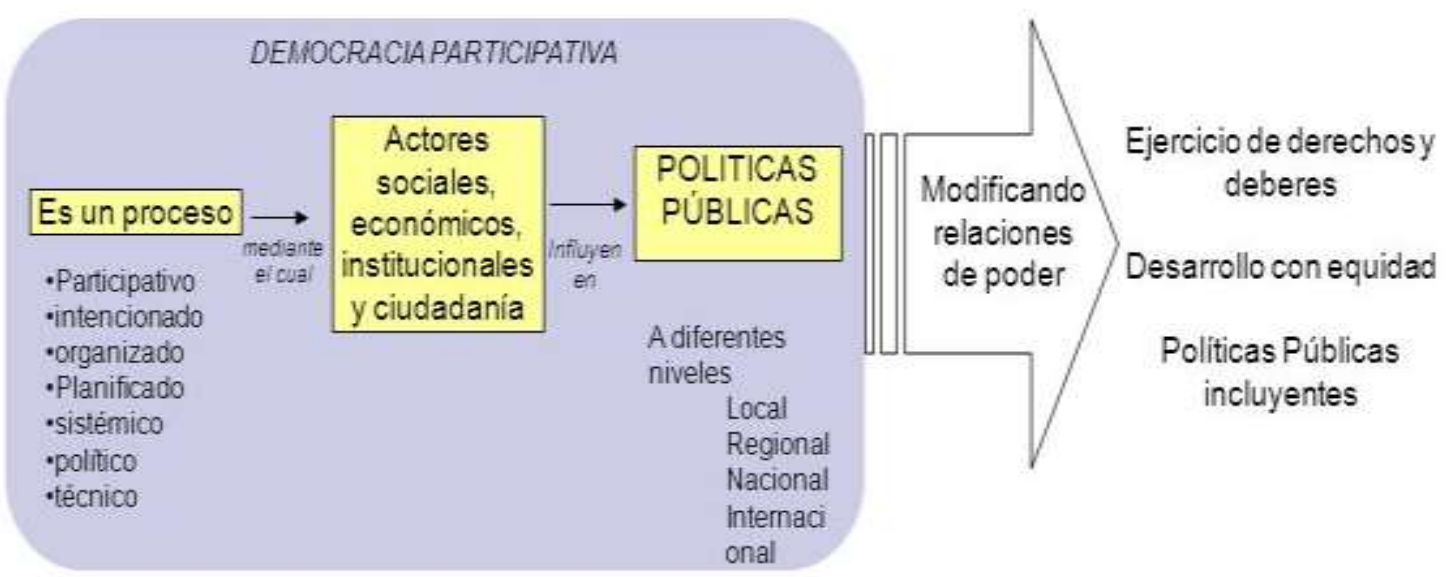

Fuente: Carreón (2016) 


\section{http://revistainvestigacionacademicasinf rontera.com}

En el proceso de gobernanza, la participación civil orientada hacia la incidencia de políticas públicas, entendida como el proceso mediante el cual la ciudadanía, los actores sociales, económicos e institucionales participan o influyen en la definición, gestión y control de políticas públicas generales o sectoriales, en los ámbitos locales, provincial, departamentales, nacionales e institucionales, supone crear, modificar o hacer cumplir y/o derogar políticas públicas (véase Figura 1).

Figura 2. Panorama de la seguridad pública

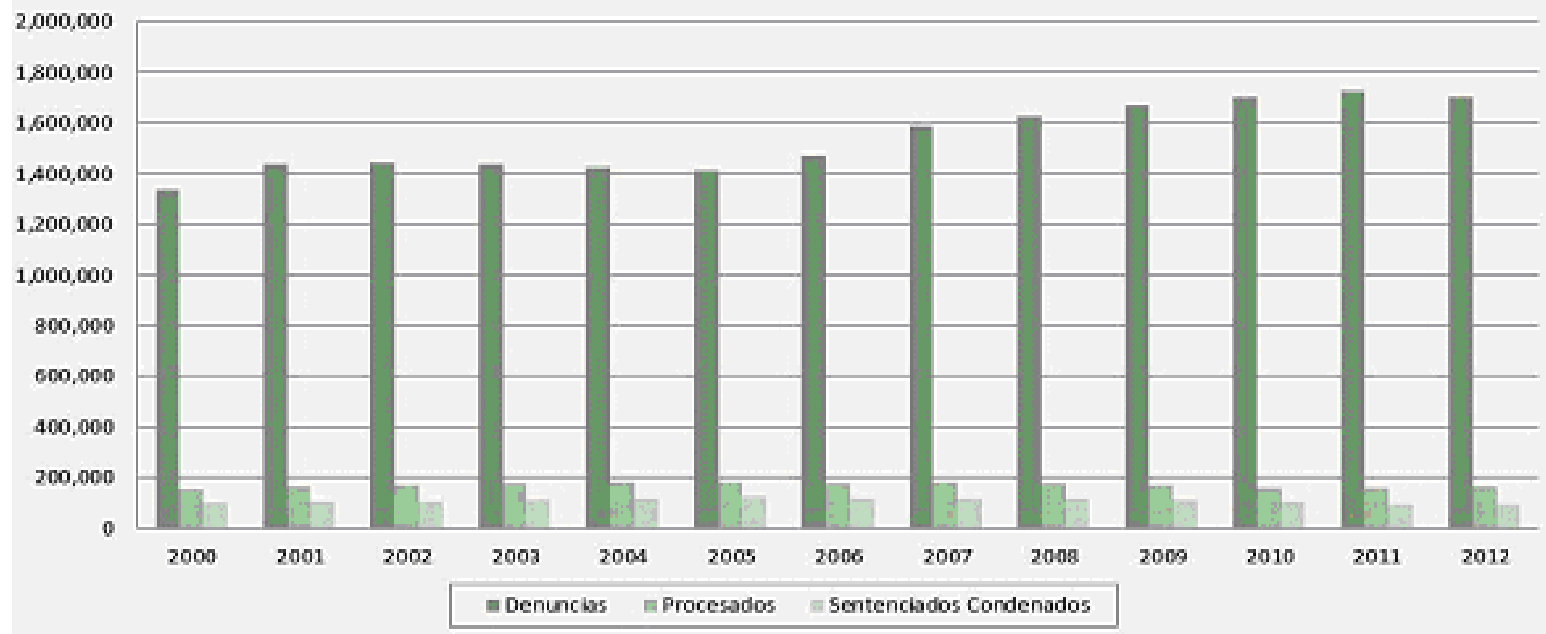

Fuente: Carreón (2016)

Sin embargo, el panorama de la seguridad dista mucho de la explicación teórica esgrimida. Indicada por niveles de comisión el delito, prevención e impartición de la justicia, es posible observar un incremento sistemático de las diferencias entre los tres rubros a favor de las denuncias respecto a casos procesados y sentenciados (véase Figura 2).

\section{Teoría de las seguridad pública}

Los marcos teóricos que explican la gobernanza de la seguridad pública advierten que las diferencias entre modelos burocráticos centrados en la denuncia y la impartición de justicia respecto a los modelos posburocráticos enfocados en la prevención del delito mediante la formación civil plantean: 1) la prevalencia de la autogestión al 


\section{http://revistainvestigacionacademicasinfrontera.com}

reducirse la función gendarme del Estado; 2) el retorno del Estado autocrático en la gestión y la administración de los procesos judiciales y 3) el establecimiento de la cogestión asumiendo una responsabilidad compartida entre los actores (véase Figura 3).

Figura 3. Teorías de la gobernanza de la seguridad pública

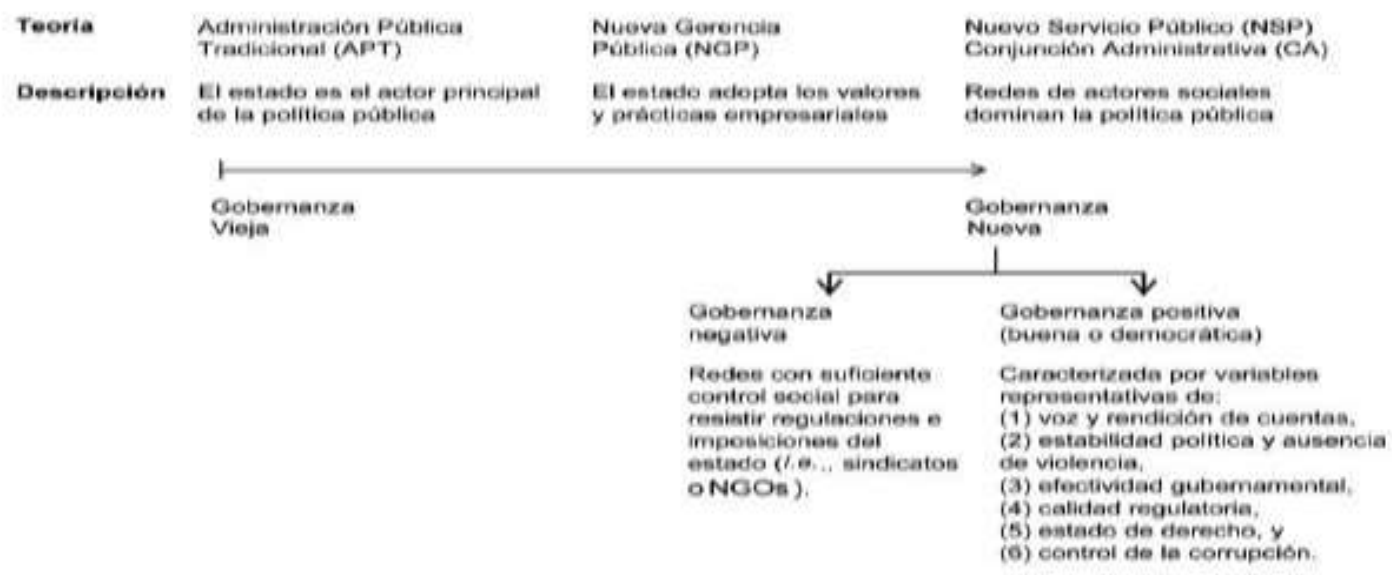

Fuente: Carreón (2016)

La teoría de la justicia que demostró las insuficiencias del intuicionismo (ideología que consideraba a las personas selectoras de lo que es bueno y lo que es malo) y la pertinencia de complementar una disyuntiva histórica: la libertad versus la igualdad para solucionar la problemática de la injusticia social generada por el Estado liberal (Carreón, García y Hernández, 2017).

La estructura social del Estado benefactor promovió la justicia social a través de instituciones que dieron por hecho una equidad social (las personas nacen con las mismas oportunidades). Esta problemática fue superada a partir de tres fundamentos (García, Carreón y Hernández, 2014):

- Toda persona posee igual derecho a la más amplia libertad compatible con una libertad igual para todos.

- Las desigualdades son inaceptables a menos que sea razonable esperar que actuarán en beneficio de todos.

- Por lo tanto, las posiciones y oficios deben estar abiertos para todos. 


\section{http://revistainvestigacionacademicasinfrontera.com}

La libertad era la conciencia de la necesidad, en consecuencia, la desigualdad implicó oportunidades exclusivas para unos cuantos individuos.

El Estado liberal como inmoral e injusto y propuso una teoría con su correspondiente método que desvanecieron y superaron una falsa dicotomía: la libertad versus la igualdad. La conciliación de ambos valores materializados en un contrato social. Se trató de la configuración de dos formas de Estado (benefactor y liberal) con base en una síntesis moral (Carreón, De la Cruz y De los Santos, 2015).

Una teoría de la justicia social y un método de equilibrio reflexivo surgieron de las problemáticas de la inmoralidad del Estado liberal mínimo. En este sentido, los valores comprensivos de tolerancia y pluralidad de visiones morales orientadas a demandas compatibles. Es en este sentido que la teoría de la justicia social estableció los fundamentos morales y jurídicos, políticos y sociales, liberales y democráticos para una sociedad democrática liberal justa. Por lo tanto, es un acercamiento interpretativo y heurístico (asume críticas y ajusta sus planteamientos) a los sistemas políticos (García et al., 2011).

Son siete los fundamentos en torno al Estado liberal justo:
- La percepción de libertad.

- Las subsiguientes creencias en torno a la equidad de oportunidades.

- Los consecuentes valores de igualdad.

- El necesario individuo moral que busca el bien social a partir de establecer un punto de equilibrio reflexivo entre los valores de la igualdad y las percepciones de libertad.

- La obvia cooperación y la posterior participación.

- La resultante sociedad democrática justa; cooperativa, ordenada y estable.

- La inevitable redistribución de la riqueza del Estado liberal para una vida plena.

El principio del maximin (elección racional de un sistema político de menores costos frente a mayores beneficios a través de un contrato social) supuso que la libertad e igualdad son principios de justicia, en un nivel institucional, fueron criterios que orientaron las decisiones ciudadanas (Carreón et al., 2013).

Los principios de igualdad tuvieron en los principios de diferencias sus contrapesos. Dichas desigualdades sociales, políticas y económicas 


\section{http://revistainvestigacionacademicasinf rontera.com}

beneficiaron a los menos aventajados. Consecuentemente, surgió la equidad (oportunidades abiertas en la obtención de oficios).

Ambos principios, develaron la improcedencia del pensamiento utilitarista que justificó las ventajas sociales, políticas y económicas en los individuos. Enmarcados por el concepto de equilibrio reflexivo entre la estructura social y las decisiones individuales, cada ciudadano acepta los dos principios de justicia para construir una sociedad ordenada con individuos moralmente libres e iguales (Carreón et al., 2014).

De este modo, el Estado fue planteado como un redistribuidor de la riqueza. A través de sus instituciones, se enfrentaría a los intereses hegemónicos que apelan al principio de libertad para lucrar con los servicios públicos. En tal sentido, la libertad de consenso como la capacidad que tienen los individuos para elegir, hacer y ser fue el principal instrumento en la concepción del Estado justo (Carreón et al., 2016).

En tal sentido, el impacto social del Estado liberal radical en relación con el Estado liberal clásico supone una forma de Estado mínimo como el único permisible y justificable para una sociedad liberal. Esta forma mínima de Estado violaba los derechos individuales. Sin embargo, en la concepción liberal, los de libre pensamiento y propiedad determinaron la función protectora del Estado. La diferencia entre las necesidades individuales coartó la cooperación entre las personas. Es decir, cada miembro autónomo, es igual en sus derechos más no en sus obligaciones de solidaridad. Moralmente, la decisión de cada individuo implicó su responsabilidad (Carreón et al., 2017).

En este sentido, el Estado benefactor al entrometerse en las libertades de los individuos afectó directa, negativa y significativamente el funcionamiento y la estructura social, los derechos políticos y mercantiles de las personas. Es así como un Estado mínimo que sólo debiera garantizar el derecho a la propiedad y la seguridad correspondiente (García et al., 2012).

Por lo tanto, el Estado liberal fue una opción pertinente porque promovió los derechos individuales. Sin embargo, históricamente el Estado ha expropiado propiedades para transferirlas a una elite. El estado ha transferido propiedades a personas particulares que no han probado merecer dichos bienes. Por lo tanto, el Estado es inmoral (García et al., 2013).

En contraste, el Estado máximo como ilegitimo por usurpador y redistribuidor. Ambas funciones le pertenecerían a la racionalidad de los individuos quienes pueden decidir cotizar, comprar, vender, intercambiar o regalar sus bienes. El Estado fue 


\section{http://revistainvestigacionacademicasinfrontera.com}

ilegitimo porque restringió estas iniciativas en las personas. Por lo tanto, la pobreza y la exclusión social, fueron consecuencias de la inmoralidad e impertinencia del Estado. Más aún, el Estado ha sido ineficaz en la distribución de la riqueza. Finalmente, la solución a la injusticia social es impulsando una nueva moralidad en la sociedad civil (García et al., 2014).

- En el Estado mínimo pueden enmarcarse las utopías de cambio moral: los derechos civiles.

- Sin embargo, bajo el Estado liberal no fue posible cambiar la moralidad humana individualista a una moralidad colectivista.

- Además, fue indispensable cambiar el marco jurídico para activar el cambio moral.

- Por lo tanto, la sociedad también debió cambiar en sus hábitos más que en sus valores.

Los conocimientos decisorios y las habilidades comunicativas implican la construcción de una entidad (Estado, grupo o individuo) moral líder. Es en las democracias gobernadas por la representación de voluntades mayoritarias y minorías proporcionales más que por oligarquías, dictaduras o tiranías, donde ambos planteamientos liberales son pertinentes. Sin embargo, esta estructura liberal democrática fue incompatible tanto para el crecimiento individual como para el desarrollo comunitario (García, 2013).

En este sentido, la igualdad de oportunidades es diferente a la igualdad de resultados. El Estado social al promover la equidad también restringe la libertad de crecimiento económico al planificar la producción y regular la oferta y la demanda (García, 2017).

\section{Estudios de las expectativas de seguridad pública}

Grosso modo, las expectativas en torno a la globalización refieren a las consecuencias esperadas ante las alianzas estratégicas entre multinacionales y pymes en torno al desarrollo local, pero su importancia radica en la exploración de climas organizacionales propicios para las redes, gestiones, producciones y transferencia de innovaciones orientadas al posicionamiento de marcas en el mercado local y su consolidación en el concierto internacional.

Las expectativas en torno a la globalización, para los fines del presente trabajo alude a las consecuencias probables de las alianzas entre multinacionales y pymes en el marco del desarrollo local. Esto es así porque las 


\section{http://revistainvestigacionacademicasinf rontera.com}

comunidades y barrios periféricos a las ciudades desarrollan una identidad semiurbana, apego al lugar y sentido de pertenencia que limita el posicionamiento de las multinacionales, pero también inhibe la emergencia de pymes dando como resultad o la alianza entre éstos dos actores hegemónicos.

Sin embargo, los estudios relativos a las alianzas estratégicas entre pymes y multinacionales advierten que ambas son orientadas por las necesidades locales, pero también por las innovaciones que permiten su inserción en el mercado local (Carreón, 2013).

De este modo es que las redes, gestiones, producciones y transferencias de innovaciones suponen activos intangibles y capitales intelectuales que ambas entidades deben sopesar a fin de poder alcanzar las ventajas competitivas que permitan el posicionamiento de sus marcas y la consolidación de sus modelos de negocios (Bustos, Quintero y García, 2017).

En tales escenarios, las percepciones de riesgo de los consumidores suponen expectativas de las consecuencias del establecimiento de alianzas estratégicas en la agenda local. Se trata de un fenómeno en el que las comunidades y barrios periféricos más bien se resisten a los estilos de vida y consumo que implican las alianzas entre multinacionales y pymes (Carreón, 2016).
La globalización es parte de un proceso en el que la localización es su complemento antagónico. Esto significa a los flujos económicos y humanos concentrados en las ciudades desarrolladas al mismo tiempo que quienes se quedan en sus lugares de origen se arraigan identificándose con el mundo global a través de los medios masivos de comunicación. Internet como la principal ventana a las imágenes y discursos, promueve ideas y sentimientos de pluralidad como libertad de elección y competencia. En contraste, en las zonas comunitarias se gestan sentimientos de solidaridad. Ambos son consecuencias de lo global y lo local que se ubican en cinco dimensiones (García, Bustos y Montero, 2012).

La globalización al ser reversión de las sociedades cerradas que aumentaron la igualdad de resultados y disminuyeron las libertades individuales por la ausencia de sus derechos y los regímenes autoritarios. Implica a un nuevo sistema social, económico y político diferente puede construirse a partir de la concatenación de sus consecuencias. Es decir, que independientemente de la igualdad y la libertad, la consecuente afectividad y racionalidad, las subsiguientes solidaridad $\mathrm{y}$ competencia, son los factores para revertir un sistema injusto para las minorías marginadas económicamente (García et al., 2011). 


\section{http://revistainvestigacionacademicasinf rontera.com}

El desequilibrio, entre la igualdad y la libertad puede revertirse a partir de sus consecuencias concentradas en la solidaridad y la competencia. Esto sugiere excluir a la burocracia estatal, permitir la auto gestión comunitaria y el desarrollo de un auto sistema educativo.

Las expectativas locales ante la globalización, indicada por las utilidades, los riesgos, las oportunidades y las capacidades esperadas, suponen un escenario de ventajas competitivas basadas en los activos intangibles y los capitales intelectuales entre los cuales destacan las expectativas como factores de anticipación a conflictos o desencuentros entre los actores económicos, políticos y sociales.

En la baraja de expectativas destacan las utilidades percibidas como factores emergentes en las alianzas estratégicas entre multinacionales y pymes, aunque su relevancia estiba en el equilibrio que suponen las demandas externas y los recursos internos en las organizaciones, su función está en el clima de tareas, los cuales determinarían el clima de innovaciones y el clima de apoyos.

Sin embargo, consustancial a las utilidades percibidas, las alianzas estratégicas entre multinacionales y pymes suponen riesgos en las transferencia de conocimiento más que en la gestión o producción de innovaciones para el mercado local. Un aumento en los riesgos no sólo implica una reducción de las utilidades esperadas sino, además implica un incremento en las capacidades ya que, en el clima de tareas donde operan los riesgos éstos se subsanan con empatía, confianza y compromiso.

Por consiguiente, el clima de relaciones prevaleciente con respecto al clima de tareas, apoyos o confianzas, abre oportunidades en la gestión y producción más que en la transferencia de información ya que, en las redes de conocimiento, los climas de tareas son hegemónicos.

Si las oportunidades emergen del clima de apoyos y tareas, entonces las capacidades se distinguirán por su grado de habilidades y conocimientos inherentes al clima de innovaciones. Esto es que las habilidades anteceden a las innovaciones, pero subyacen de las relaciones de confianza y empatía, cuando no del compromiso.

El estudio de las expectativas de las consecuencias del proceso globalizador centrado en las alianzas estratégicas entre multinacionales y pymes supone la observación de las oportunidades y capacidades de gestión del conocimiento más que de producción y transferencia.

Sin embargo, un escenario que se distingue por alto grado de relaciones de empatía, confianza y 


\section{http://revistainvestigacionacademicasinf rontera.com}

compromiso más que por el cabal funcionamiento de sus estructuras de gestión, producción y transferencia de conocimiento implica más expectativas de riesgo que expectativas de beneficio.

Hernández et al., (2017), Limón et al., (2017), Sánchez et al., (2017) y Sandoval et al., (2017) resaltan a la identidad como el denominador común entre los desequilibrios que propician las multinacionales en las pymes, valores y normas locales, las expectativas se centren en un tipo de identidad resultante del clima organizacional más que del contexto en el que se enmarca.

Sin embargo, un estudio comparativo entre localidades con y sin alianzas estratégicas de multinacionales con pymes permitirá establecer diferencias significativas si las hubiera, o bien, establecer la incidencia de los actores económicos y políticos sobre los actores sociales, principalmente en los talentos, activos intangibles y capitales intelectuales.

Formulación: ¿Los marcos teóricos relativos a la seguridad pública en el ámbito organizacional (en adelante seguridad organizacional) ala sumir que la comisión de un delito puede ser observado y comparado con otros casos y en consecuencia, anticipar sus efectos y reducir su incidencia en la dinámica empresarial explicarán el fenómeno en un contexto local donde prevalece un alto índice de asaltos, secuestros, extorsiones, lesiones y fraudes a microempresarios?

Hipótesis de simplicidad: Las teorías que explican los ejes y trayectorias de discusión en torno a la seguridad organizacional advierten que es posible observar y predecir el fenómeno en contextos locales de alta corrupción: delitos, impunidad, negligencia, opacidad y nepotismo.

Hipótesis de complejidad: No obstante que los marcos teóricos han explicado y anticipado las problemáticas delictivas asociadas a la corrupción, la especificidad de los casos locales supone que estamos ante eventos inciertos en torno a los que no es posible explicar ni predecir los riesgos y las amenazas que supone una estructura de corrupción en todos los niveles de gobierno, así como el grado de colusión con la delincuencia local e internacional en detrimento de la dinámica empresarial.

\section{Método}

En un primer estudio ee realizó la especificación de un modelo para su estudio a partir de un diseño no experimental, transversal y exploratorio con una selección muestral no probabilística de fuentes indexadas a repositorios nacionales, considerando el año de publicación de 2000 a 2017, así como la inclusión de conceptos tales como "globalización", "localidad" y "modelo". 


\section{http://revistainvestigacionacademicasinf rontera.com}

A partir de la técnica Delphi, la cual consiste en comparar la información e integrar los datos para establecer los ejes y las trayectorias de relaciones entre los factores esgrimidos en la literatura consultada. Se advierten líneas comparativas entre entidades $\sin$ o con alianzas estratégicas de multinacionales con pymes a fin de poder establecer diferencias y anticipar la innovación del conocimiento.

En un segundo estudio se llevó a cabo un estudio exploratorio, transversal de corte cualitativo y cuantitativo. Se realizó una selección no probabilística de 258 microempresarios. El criterio de selección fue pertenecer al sistema de prácticas y servicio social en organizacionales e instituciones con y sin fines de lucro del municipio de Chimalhuacán, Estado de México.

Se utilizó la Escala de Expectativas de la Seguridad Organizacional de Carreón (2014) la cual incluye 15 aseveraciones en torno a tres dimensiones relativas a los riesgos y beneficios de invertir en seguridad reclutadora, tecnológica e interpersonal. Cada ítem se responde con alguna de cinco opciones que van desde $0=$ nada probable, 1 = muy poco probable, $2=$ poco probable, 3 = moderadamente probable, $4=$ muy probable, 5 = bastante probable.
Se encuestaron a los participantes a través de sus correo electrónicos, previo contacto telefónico. Se les informó que los resultados de la investigación no afectarían ni positiva ni negativamente su situación escolar. Se les pidió que contestaran honestamente a las preguntas y aseveraciones. Se les invitó a que consultaran los resultados en el informe final del grupo de investigación. Los datos fueron procesados en el Paquete Estadístico para Ciencias Sociales (SPSS por sus siglas en inglés) y el Software de Análisis de Momentos Estructurales (AMOS por sus siglas en inglés) en sus versiones 10 y 6.0

Se utilizaron los parámetros de curtosis, alfa de Cronbach, coeficientes KMO, prueba de Bartlett, pesos factoriales, correlaciones de Pearson, covarianzas "phi", pesos "beta" y "gama", así como índices de ajuste $\mathrm{y}$ residuales a fin de contrastar el modelo de relaciones especificadas con los datos observados.

\section{Resultados}

La Tabla 1 muestra las propiedades psicométricas del instrumento que mide las tres dimensiones relativas a los riesgos y beneficios de invertir en seguridad empresarial, tecnológica y personal, las cuales superaron el nivel de consistencia requerido (alfa de 0,700).

Tabla 1. Descriptivos del instrumento 
Julio- Diciembre 2017

"Eloskes de nis lija.

Revista de Investigación

Año 10.

Académica sin Frontera

Núm. 26

ISSN: 2007-8870

http://revistainvestigacionacademicasinfrontera.com

\begin{tabular}{|c|c|c|c|c|c|c|c|}
\hline Código & Ítem & $M$ & $D E$ & $\boldsymbol{A}$ & F1 & $F 2$ & F3 \\
\hline & Subescala de expectativas de seguridad reclutadora & & & 0,780 & & & \\
\hline SE1 & Emitiré credenciales para identificar a los empleados & 3,20 & 1,01 & 0,761 & & & 0,326 \\
\hline SE2 & Investigaré a los empleados antes de contratarlos & 3,24 & 1,02 & 0,751 & & & 0,346 \\
\hline SE3 & Revisaré las cartas de recomendación antes de hacer contrataciones & 3,56 & 1,04 & 0,750 & & & 0,329 \\
\hline SE4 & Integraré los expedientes de empleados para conocer su perfil & 3,82 & 1,05 & 0,7663 & & & 0,310 \\
\hline \multirow[t]{2}{*}{ SE5 } & Visitaré las casas de los futuros empleados antes de contratarlos & 3,01 & 1,02 & 0,703 & & & 0,305 \\
\hline & Subescala de seguridad tecnológica esperada & & & 0,777 & & & \\
\hline ST1 & Instalaré videocámaras en cada rincón de la empresa & 3,82 & 1,05 & 0,701 & & 0,317 & \\
\hline ST2 & Instalaré micrófonos para escuchar las conversaciones de empelados & 3,42 & 1,08 & 0,762 & & 0,305 & \\
\hline ST3 & Instalaré detectores de metales en las entradas de la empresa & 3,47 & 1,02 & 0,750 & & 0,304 & \\
\hline ST4 & Instalaré alarmas conectadas a la estación de policía & 3,56 & 1,09 & 0,753 & & 0,392 & \\
\hline \multirow[t]{2}{*}{ ST5 } & Instalaré un cuarto de pánico en las oficinas de la empresa & 3,06 & 1,02 & 0,701 & & 0,301 & \\
\hline & Subescala de expectativas de seguridad interpersonal & & & 0,765 & & & \\
\hline SP1 & Contrataré guardaespaldas para mi seguridad personal & 3,26 & 1,01 & 0,752 & 0,307 & & \\
\hline SP2 & Contrataré guardaespaldas para la seguridad de mi pareja & 3,24 & 1,03 & 0,755 & 0,306 & & \\
\hline SP3 & Contrataré guardaespaldas para mi familia & 3,56 & 1,05 & 0,753 & 0,391 & & \\
\hline SP4 & Contrataré guardaespaldas para mis socios & 3,20 & 1,08 & 0,750 & 0,382 & & \\
\hline SP5 & Contrataré guardaespaldas para mis talentos & 3,21 & 1,05 & 0,749 & 0,302 & & \\
\hline
\end{tabular}

Método de extracción: ejes principales, rotación promax. Adecuación y esfericidad $\left\{\mathrm{X}^{2}=234,35(12 \mathrm{gl}) \mathrm{p}=0,000 ; \mathrm{KMO}=0,756\right\rfloor \mathrm{M}=$ Media, DE = Desviación Estándar, A = Alfa de Crombach quitando el valor del ítem, F1 = Expectativas de Seguridad Reclutadora (34\% de la varianza total explicada y alfa de 0,780), F2 = Expectativas de Seguridad Tecnológica (22\% de la varianza total explicada y alfa de 0,777), F3 = Expectativas de Seguridad Interpersonal (18\% de la varianza total explicada y alfa de 0,765$)$. Todos los ítems se responden 


\section{http://revistainvestigacionacademicasinf rontera.com}

con alguna de cinco opciones: $0=$ nada probable, $1=$ muy poco probable, $2=$ poco probable, $3=$ moderadamente probable, $4=$ muy probable, $5=$ bastante probable.

El total de varianza explicada del constructo fue de $74 \%$, lo cual denota un alto porcentaje de explicación del fenómeno de la seguridad organizacional, razón por la cual se contrastó un modelo de relaciones reflejantes entre ítems y los factores (véase Figura 4).

Figura 4, modelo estructural reflejante

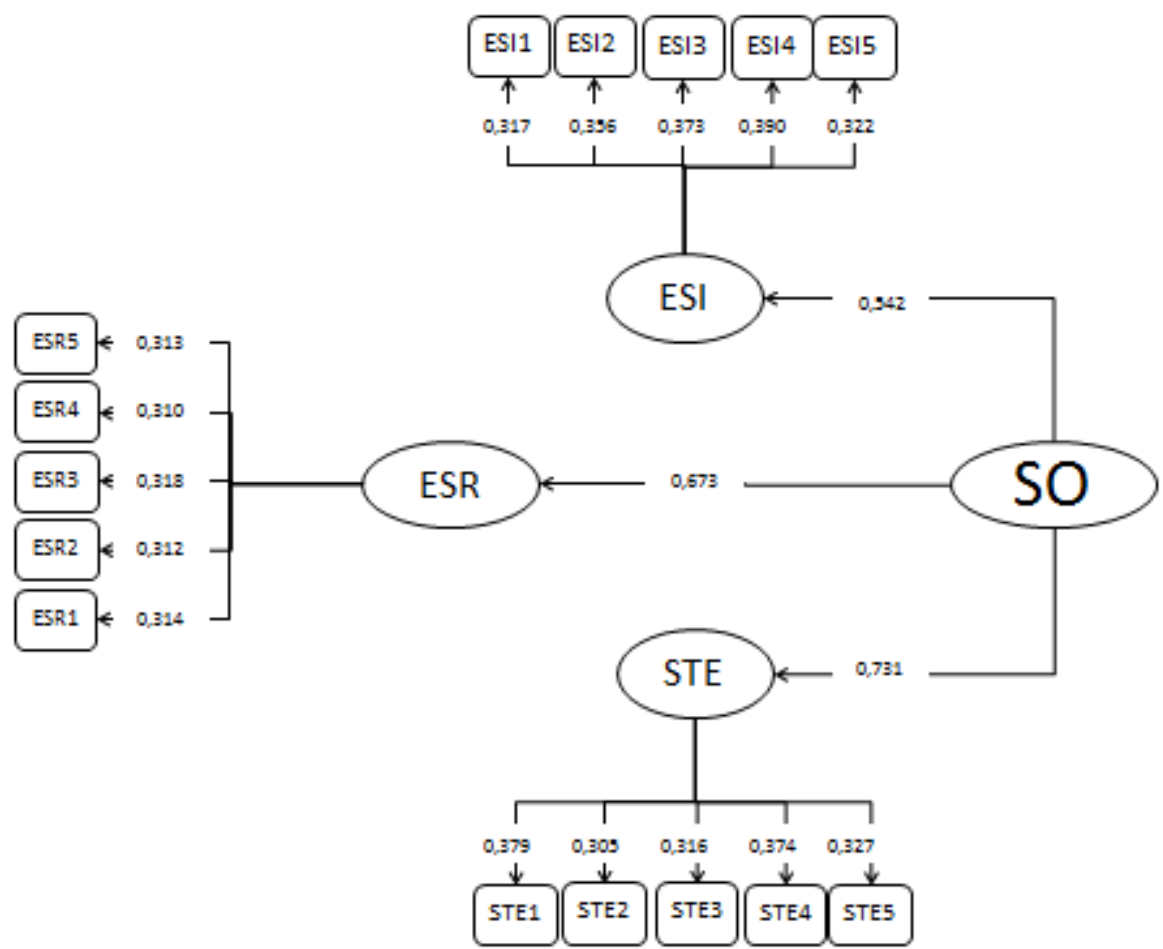

El modelo estructural incluye las asociaciones entre los tres factores y con respecto a cada uno de los ítems, es posible inferir que se trata de un constructo de tres dimensiones por el alto porcentaje de varianza que explica, aunque está reflejado por el factor de seguridad tecnológica esperada $(0,731)$. La aceptación de la hipótesis nula se dedujo de los parámetros de ajuste y residual $\left\{\mathrm{X}^{2}=324,35(21 \mathrm{gl}) \mathrm{p}=0,007\right.$; GFI $=$ 0,$990 ; \mathrm{CFI}=0,995 ; \mathrm{RMSEA}=0,009 \mathrm{~J}$

\section{Discusión}

El aporte del presente trabajo al estado de la cuestión estriba en la especificación de un modelo 


\section{http://revistainvestigacionacademicasinf rontera.com}

estructural explicado por tres factores hasta en un $77 \%$, pero el tipo de análisis estadístico limita los resultados a la muestra de estudio, sugiriendo el contraste del modelo a otros escenarios de estudio como es el caso de las organizaciones con fines de lucro en latitudes con niveles de corrupción equiparables al centro de México.

No obstante que el modelo es en su mayoría explicado por los tres factores, habría la posibilidad de incluir un cuarto factor considerando los protocolos financieros donde los fraudes, el robo de identidad y la violación a la privacidad merman a las organizaciones en cuanto a sus finanzas.

De este modo, el trabajo de Carreón et al., (2017) advierte que el ciber-espionaje ha repercutido en las organizaciones dedicadas a la creación del conocimiento, siendo que en su proceso de gestión contrarrestan sus ventajas competitivas con software espías que develan la innovación de sus procesos.

En tal sentido, el presente trabajo ha demostrado que es la dimensión tecnológica de la seguridad el factor que refleja a la seguridad organizacional, pero su especificidad en las transferencias y protocolos digitales permitirá explicar el restante $23 \%$ de la varianza total no explicada del constructo.
Sánchez et al., (2017) encontraron que los procesos innovadores y los protocolos de seguridad son consustanciales a la dirección estratégica ya que, si bien un clima de relaciones explica la productividad y la competitividad a través del compromiso, es el clima de innovación en que determina la formación profesional de talentos dedicados a la innovación de los procesos y el manejo de sus controles de seguridad.

El presente estudio, acorde con la relación entre adquisición de la tecnología y procesos de innovación encontró una estructura factorial orientada por tres factores que reflejan un constructo relativo a la seguridad organizacional, pero deslindada de una formación profesional responsable como sería la prevención del delito al interior de la empresa.

Se trata de un factor que también podría explicar el restante $23 \%$ de la varianza ya que, es la empresa la encargada de formar a sus recursos cuando éstos se comprometen a un mayor desempeño incentivados o no por recompensas y reconocimientos.

Desde la óptica del clima laboral, la formación profesional libre de violencia con orientación a valores de respeto, equidad y solidaridad serían un dispositivo de prevención eficaz del delito en las organizaciones dedicadas a la creación del 


\section{http://revistainvestigacionacademicasinf rontera.com}

conocimiento y por ende a la vigilancia de sus procesos de calidad e innovación.

Pues bien, si el compromiso laboral está relacionado con la seguridad organizacional, entonces será la formación profesional, la capacitación y el adiestramiento los pilares de la prevención del delito al interior de las organizaciones, planteando una gestión integral del conocimiento respecto a la seguridad instrumentada por alguna tecnología de videovigilancia, detección de metales o anti software espías.

\section{Conclusión}

El objetivo del presente trabajo ha sido establecer la consistencia interna de un modelo a fin de poder validar su estructura y especificarla para contrastarla en otros escenarios similares al que prevalece en el centro de México.

La pregunta de investigación se circunscribió a la pertinencia de la explicación universal sobre la problemática de seguridad local, pero la hipótesis aceptada sugirió que los marcos teóricos no sólo explican al fenómeno y más bien lo predicen.

En tal sentido es que el estudio de la seguridad organizacional deberá transitar por el contraste de modelo que por su grado de porcentaje de varianza explicada incluirá otros factores relativos a la seguridad informática, digital o electrónica que inhibe los procesos innovadores en las organizaciones dedicadas a la creación del conocimiento.

\section{Agradecimientos}

El proyecto fu financiado por la Universidad nacional Autónoma de México, Dirección General de Asuntos del Personal Académico, Programa de Apoyo a Proyectos de Investigación e Innovación Tecnológica, registro número IN305566.

\section{Referencias}

1. Bustos, J. M., Quintero, M. L. y García, C. (2017). Gobernanza de la sustentabilidad local: Índice de mediatización hídrica para la Ciudad de México. Tlatemoani, 24, 143-159

2. Carreón, J. (2013). Discursos en torno a la migración laboral, el retorno y la reinserción social a partir de la identidad grupal en Xilitla, micro-región de la Huasteca Potosina (México). en L. Cano (coord.). Pobreza y desigualdad social. Retos para la reconfiguración de la política social. (pp. 153-174). México: UNAM-ENTS 


\section{http://revistainvestigacionacademicasinf rontera.com}

3. Carreón, J. (2016). Desarrollo humano: Gobernanza y emprendimiento social. México: UNAM-ENTS

4. Carreón, J., De la Cruz, P. I. y De los Santos, M. (2015). La administración de temores sociales. Contrastes de un modelo de percepción de la inseguridad en México. Ehquidad, 4, 31-60

5. Carreón, J., García, C. y Hernández, J. (2017). Las situaciones hídricas, los estilos de vida en las zonas urbanas periféricas y las luchas por el agua. En M. E. Alvarado (coord.). experiencias universitarias de los movimientos sociales y la acción colectiva. Una mirada al pasado y al futuro desde el presente. (pp. 105-128). México: UNAM-CEIICH

6. Carreón, J., García, C., Morales, M. y Rosas, F. (2013). Compromiso laboral en el Trabajo Social del sector salud. Invurnus, 8 (2), 15-23

7. Carreón, J., Hernández, J. y Quintero, M. L. (2016). Especificación de un modelo de desarrollo local. En D. Del Callejo, Canal, M. E. y Hernández, G. (coord.). orientaciones metodológicas para el estudio del desarrollo. (pp. 149-168). México: Universidad Veracruzana.
8. Carreón, J., Hernández, J., Bustos, J. M. y García, C. (2017). Políticas de fomento empresarial y sus efectos sobre las percepciones de riesgo en caficultores de Xilitla, san Luis Potosí, centro de México. Poiesis, 32, 33-51

9. Carreón, J., Hernández, J., Quintero, M. L., García, C. y Mejía, S. (2016). Redes de conocimiento en torno a la complejidad organizacional: aprendizaje de la autoregulación, disipación, adaptabilidad y dinamismo ante los cambios. Prospectiva, 2 (2), 57-70

10. Carreón, J., Morales, M., Rivera, B., García， C. y Hernández, J. (2014). Emprendedurismo migrante y comerciante: Estado del conocimiento. Tlatemoani, 15, 1-30

11. García, C. (2013). La inseguridad migratoria en los medios impresos. Reflexiones, 92, 159-173

12. García, C. (2017). Los estudios psicológicos del establecimiento de agenda: especificación y discusión de un modelo de identidad conformista versus cooperativista. Nómadas, 50, 1-18

13. García, C., Bustos, J. M. y Montero, M. (2012). Dimensiones de la sustentabilidad hídrica. En J. Carreón, y 


\section{http://revistainvestigacionacademicasinfrontera.com}

Hernández, J. 8coord). Psicología de la globalización neoliberal: análisis de la exclusión social, la brecha digital y el desarrollo sustentable. (pp. 117-172).

México: Díaz de Santos

14. García, C., Bustos, J. M., Juárez, M., Rivera, B. L. y limón, G. A. (2017). Expectativas de usuarios del servicio de agua potable en torno al abastecimiento, la calidad y las tarifas en el marco de futuras elecciones en una localidad de la Ciudad de México. Compendium, 4 (7), $35-54$

15. García, C., Carreón, J. y Bustos, J. (2012). Socialización y compromiso laboral en trabajadoras sociales de instituciones públicas y privadas. Humanismo y Trabajo Social, 11, 177192

16. García, C., Carreón, J. y Hernández, J. (2014). La formación profesional del capital humano en la civilización del cambio climático. Revista Internacional de Investigación en Ciencias Sociales, 10 (1), 107-125

17. García, C., Carreón, J., Hernández, J. y Bustos, J. (2015). Gobernanza del riesgo desde la percepción de amenazas y el sentido de comunidad. En S. Vázquez., Cid, B. y Montemayor, E. (coord.). Riesgos y Trabajo Social. (pp. 71-94). México: UAT
18. García, C., Carreón, J., Hernández, J. y Bustos, J. M. (2014). Estructura de las percepciones de riesgo en torno a la escasez y el desabasto de agua global y local. En J. M. Bustos y Flores, L. M. (coord.). Psicología ambiental, análisis de barreras y facilidades psicosociales para la sustentabilidad. (pp. 105-118). México: UNAM-FES Zaragoza

19. García, C., Carreón, J., Hernández, J. y Bustos, J. M. (2016). Gobernanza del riesgo desde la percepción de amenazas y el sentido de la comunidad. En S. Vázquez, Cid, B. G., Montemayor, E. (coord.). Riesgos y trabajo social. (pp. 71-94). México: UAT

20. García, C., Carreón, J., Hernández, J. y Morales, M. (2014). Contraste de un modelo de compromiso laboral en centros de salud pública. Acta Universitaria, 24 (1), 48-59

21. García, C., Carreón, J., Hernández, J. y Salinas. (2016). Gobernanza de los actores y redes de innovación tecnológica. En M. L. Quintero, Sales, J. y Velázquez, E. B: (coord.). Innovación y tecnología. Retos para su aplicación práctica en las empresas. (pp. 79-94). México: Porrúa-UAEMEX UAP Nezahualcóyotl 


\section{http://revistainvestigacionacademicasinf rontera.com}

22. García, C., Carreón, J., Hernández, J., Bautista, M. y Méndez, A. (2012). Modelamiento de variables sociopsicorganizacionales a partir de la revisión del estado del arte. Revista de la Facultad de Trabajo Social, 28 (28), 1360

23. García, C., Carreón, J., Hernández, J., Bustos, J. y Aguilar, J. (2015). Especificación de un modelo de hipermetropía sociopolítica. Luna Azul, $42,270-292$

24. García, C., Carreón, J., Hernández, J., Carbajal, C., Quintero, M. L., Sandoval, F. R. y Valdés, O. (2014). Incidencia de las políticas de micro-financiamiento sobre la percepción de emprendimiento caficultor e implicaciones para el Trabajo Social. Equidad, 6, 11-36

25. García, C., Carreón, J., Hernández, J., Limón, G. A. y Morales, M. L. (2014). Los determinantes de la preocupación hidrológica en Iztapalapa. En J. M. Bustos y Flores, L. M. (coord.). Psicología ambiental, análisis de barreras y facilidades psicosociales para la sustentabilidad. (pp. 93-104). México:

UNAM-FES Zaragoza

26. García, C., Carreón, J., Hernández, J., Montero, M. y Bustos, J. M: (2012).
Sistemas de complejidad política.

Trabajo Social Hoy, 65, 39-48

27. García, C., Carreón, J., Mecalco, J., Hernández, J., Bautista, M. y Méndez, A. (2013). Sistemas políticos complejos: Implicaciones para la seguridad pública sustentable. Acciones e Investigaciones Sociales, 34,186-216

28. García, C., Valdés, O., Sánchez, R., Elizarraráz, G., Méndez, A. y Hernández, J. (2011). Diferencias entre emprendedores internautas con respecto a empatía, percepciones de riesgo y uso de aplicaciones tecnológicas. Prospectiva, $12(1), 68-75$

29. Hernández, J., Carreón, J., García, C. Aguilar, J. A. y López, L. R. (2017). Confiabilidad y validez de un instrumento que mide actitudes hacia la sustentabilidad. En M. L. Quintero, Velázquez, E. B. y Sales, J. (coord.). Enfoques, estrategia y transdisciplina en los estudios de la sustentabilidad. (pp. 95-110). México: UAEMEX-UAPN

30. Limón, G. A., Rivera, B. L., Bustos, J. M., Juárez, M. y García, C. (2017). Gobernanza del cambio climático: especificación de un modelo de actitudes 
Núm. 26

ISSN: 2007-8870

\section{http://revistainvestigacionacademicasinf rontera.com}

hacia los gobiernos del cambio climático.

Ciencia Política, 30, 1-31

31. Sánchez, A., Quintero, M. L., Sánchez, R., Fierro, E. y García, C. (2017). Gobernanza del emprendimiento social: especificación de un modelo para el estudio de la innovación local. Nómadas, $51,1-21$

32. Sandoval, F. R., Carreón, J., García, C., Quintero, M. L. y Bustos, J. M. (2017). Modelo de los determinantes de la percepción de resiliencia a partir del riesgo y estrés percibidos en relación con la gobernanza de la protección civil. Invurnus, 12 (1), 30-35

33. Sandoval, F., García, H. y Pérez, A. (2015). Género y resiliencia ante las catástrofes ambientales. Revista Iberoamericana de Producción Académica y Gestión Educativa, 3, 1-20 